\title{
Redundant nerve roots in lumbar spinal stenosis: inter- and intra-rater reliability of an MRI-based classification
}

\author{
Luca Papavero $^{1}$ (1) Carlos J. Marques ${ }^{2,3}$ (D) $\cdot$ Jens Lohmann ${ }^{1} \cdot$ Thies Fitting $^{4} \cdot$ Kathrin Schawjinski $^{1} \cdot$ Nawar Ali $^{1}$. \\ Hauke Hillebrand ${ }^{1}$ (D) $\cdot$ Rainer Maas $^{5}$
}

Received: 25 October 2019 / Accepted: 4 December 2019 / Published online: 14 December 2019

(C) The Author(s) 2019

\begin{abstract}
Purpose Patients with central lumbar spinal stenosis (LSS) have a longer symptom history, more severe stenosis, and worse postoperative outcomes, when redundant nerve roots (RNRs) are evident in the preoperative MRI. The objective was to test the inter- and intra-rater reliability of an MRI-based classification for RNR.

Methods This is a retrospective reliability study. A neuroradiologist, an orthopedic surgeon, a neurosurgeon, and three orthopedic surgeons in-training classified RNR on 126 preoperative MRIs of patients with LSS admitted for microsurgical decompression. On sagittal and axial T2-weighted images, the following four categories were classified: allocation (A) of the key stenotic level, shape (S), extension (E), and direction (D) of the RNR. A second read with cases ordered differently was performed 4 weeks later. Fleiss and Cohen's kappa procedures were used to determine reliability.

Results The allocation, shape, extension, and direction (ASED) classification showed moderate to almost perfect inter-rater reliability, with kappa values $(95 \% \mathrm{CI})$ of $0.86(0.83,0.90), 0.62(0.57,0.66), 0.56(0.51,0.60)$, and $0.66(0.63,0.70)$ for allocation, shape, extension, and direction, respectively. Intra-rater reliability was almost perfect, with kappa values of 0.90 $(0.88,0.92), 0.86(0.84,0.88)$, and $0.84(0.81,0.87)$ for shape, extension, and direction, respectively. Intra-rater kappa values were similar for junior and senior raters. Kappa values for inter-rater reliability were similar between the first and second reads $(p=0.06)$ among junior raters and improved among senior raters $(p=0.008)$.

Conclusions The MRI-based classification of RNR showed moderate-to-almost perfect inter-rater and almost perfect intra-rater reliability.
\end{abstract}

Keywords Redundant nerve roots · Lumbar spinal stenosis · Neurogenic claudication · Magnetic resonance imaging · Classification

LP and CJM contributed equally to this work and are joint first authors.

Carlos J. Marques

cmarques@schoen-klinik.de

1 Clinic for Spine Surgery, Schoen Clinic Hamburg Eilbek, Academic Hospital of the University of Hamburg, Dehnhaide 120, 22081 Hamburg, Germany

2 Science Office of the Orthopedic and Joint Replacement Department, Schoen Clinic Hamburg Eilbek, Dehnhaide 120, 22081 Hamburg, Germany

3 Non-Medical PhD Program, Faculty of Medicine, University of Hamburg, Hamburg, Germany

4 Department of Radiology at the Schoen Clinic Hamburg Eilbek, Academic Hospital of the University of Hamburg, Dehnhaide 120, 22081 Hamburg, Germany

5 Radiology Office Raboisen 38, Hamburg, Germany

\section{Introduction}

Decompression treatment for lumbar spinal stenosis (LSS) is the most common spine surgery in patients older than 65 years in the USA [1]. In roughly $60 \%$ of patients with LSS scheduled for surgery, the natural course of cauda nerve roots (CNR) remains unaltered, even in the presence of severe stenosis (Fig. 1). In the remaining $40 \%$ of patients, redundant nerve roots (RNRs) of the cauda equina are evident on preoperative magnetic resonance images (MRIs) [2-5]. RNRs were first described by Verbiest [6] in 1954, and they were named 14 years later by Cressman and Pawl [7]. RNRs were described as thickened, buckling, coiled CNRs with a serpentine (Fig. 2a) or loop-like (Fig. 2b) shape in sagittal T2-weighted images (T2WI) [8].

Little is known about the clinical significance of RNRs in patients with LSS. A recent meta-analysis revealed that, 


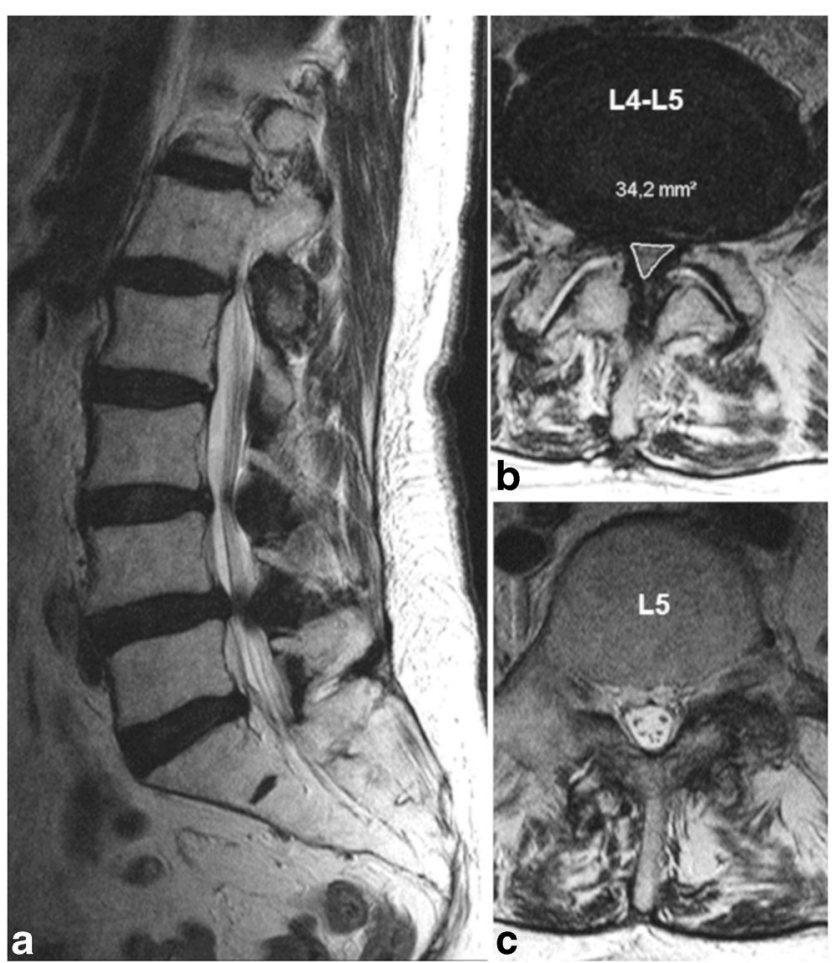

Fig. 1 a Sagittal T2-weighted images (WI) with an almost normal course of the cauda nerve roots (CNRs) despite a stenotic level grade D at L4/L5 (b) according to Schizas et al. [17] in the axial slice. c The CNRs are distributed throughout the cross-sectional area of the dural sac (positive nerve roots sedimentation sign). No evidence of redundant nerve roots (RNR-)

among patients with LSS, those that showed evidence of RNRs were older, had a longer symptom history, and presented higher degrees of lumbar stenosis preoperatively than those without RNRs. Moreover, after decompression surgery, patients with RNRs showed worse clinical scores and lower recovery rates than those without RNRs [9]. A study on potential RNR predictors demonstrated that patients with LSS with evidence of RNRs on the preoperative MRI were older had a shorter lumbar spine canal, displayed stenosis at more levels, and had more severe stenosis, compared to patients without RNRs [10].

Yokoyama et al. studied patients with LSS and found that most RNRs resolved postoperatively, but some did not. Among patients with unresolved postoperative RNRs, functional outcome remained poor, even when the dural sac was sufficiently expanded. Furthermore, among patients with LSS, those with loop-shaped RNRs performed worse than those with serpentine-shaped RNRs [11].

Although the etiology and pathogenesis of RNRs are only partially understood, they appear to indicate more advanced LSS stages, and they are a negative prognostic factor [9].

In daily radiological practice, MRI reports of patients with LSS mostly describe changes in bony structures, discs, facet joints, and yellow ligament. A validated classification system for RNRs could facilitate descriptions of changes in the CNR

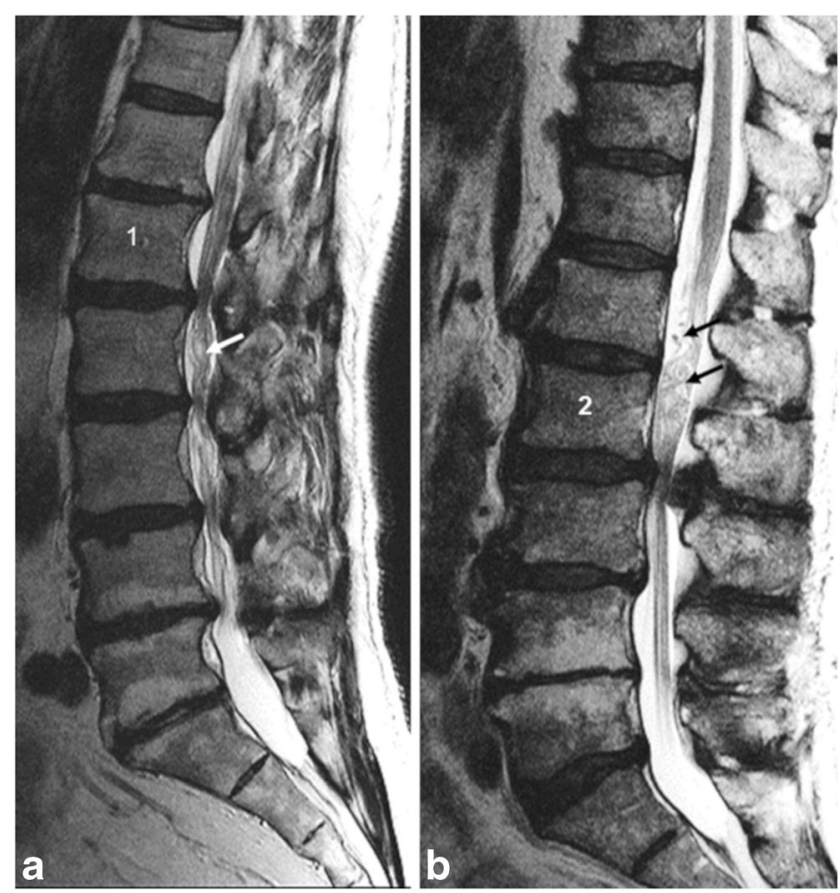

Fig. 2 a Sagittal T2WI with stretched cauda nerve roots cranially and serpentine RNRs (white arrow) caudally from the key stenotic level (KSL) at L1/L2. b KSL at L2/L3 with stretched CNRs caudally and loop-shaped RNRs cranially (black arrows)

and could provide clinicians with additional relevant information. To the best of our knowledge, a validated classification system for RNRs does not exist [12]. The aim of the present study was to test the inter-rater and intra-rater reliability of an MRI-based classification system for RNRs in LSS.

\section{Materials and methods}

\section{Study design}

For this retrospective study, we acquired database data to investigate inter- and intra-rater reliability. This study was developed in accordance with the "Guidelines for Reporting Reliability and Agreement Studies" (GRRAS) [13]. The reporting follows the STROBE Statement guidelines [14].

The Ethics Commission of the Federal State of Hamburg, Germany, approved the research proposal (File PV 5767). Informed consent was not necessary, because the data were collected and treated anonymously.

\section{Sample}

A sample size calculation was based on the work by Rotondi and Donner [15]. First, we assumed the proportions of the three items in the category "direction" were $0.10,0.20$, and 0.70 . We determined that $126 \mathrm{MRIs}$ were required to ensure that a two-sided $95 \%$ 
confidence interval (CI) for a target kappa value $(k)$ of 0.80 did not exceed the lower bound of 0.70 .

We identified data for 126 patients with LSS (47 females) that underwent decompression surgery. The mean age was $74.2 \pm 9$ years. Women (mean age 76.4 \pm 8.9 years $)$ were 3.4 years older $(P=0.03)$ than men (mean age $72.9 \pm 8.9$ years). All patients had evidence of RNRs and underwent decompression surgery in the same institution, between December 2012 and August 2016.

Inclusion criteria were as follows: symptomatic central LSS that required surgical decompression without fixation; available preoperative MRIs of at least 1.5 Tesla (T), including sagittal and axial T2-WIs, stored in the picture archive and communication system of the institution; and evidence of RNRs. Exclusion criteria were as follows: a previous history of lumbar spine surgery; scoliosis or a vertebral slip that required fixation; and congenital, traumatic, infectious, or neoplastic diseases of the lumbar spine.

\section{The raters}

Three senior raters (one neuroradiologist, one orthopedic surgeon, and one neurosurgeon with 15, 10, and 35 years of experience, respectively) and three junior raters (orthopedic surgeons in training) independently classified all RNRs on the MRIs.

Fig. 3 a Sagittal T2WI with RNRs cranial, b caudal, and c cranial-caudal from the KSL. The ASED notation would be as follows: a RNR+: L2/L3.S.1+.cr; b RNR+: L4/L5.L.1.ca; and c RNR+: L3/L4.L.1+.cc

\section{The MRI-based definition of RNRs}

An MRI was defined as presenting RNRs when the key stenotic level (KSL) altered the natural course of the CNR. In most cases, CNRs were straight on one side of the KSL and serpentine or loop-shaped on the opposite side. Rarely, the CNR looked either serpentine or coiled on both sides of the KSL. Most RNRs were located cranial to the KSL, some were located caudal to the KSL, and a few were located cranialcaudal to the KSL [16] (Fig. 3).

\section{The ASED classification of RNRs}

The morphologies of RNRs were classified into four categories, including allocation, shape, extension, and direction (ASED). The ASED classification system is described in Table 1. Examples are illustrated in Fig. 3.

\section{Procedures}

The classifications of shape, extension, and direction depend on the allocation. Therefore, all raters first classified the allocations independently. Then, we calculated the inter-rater kappa value for allocation. In 22 cases, discordances occurred between at least two raters. These cases were discussed and resolved by consensus. Thereafter, all raters independently classified the RNRs on the 126 MRIs, according to the definitions for shape, extension, and direction, considering the
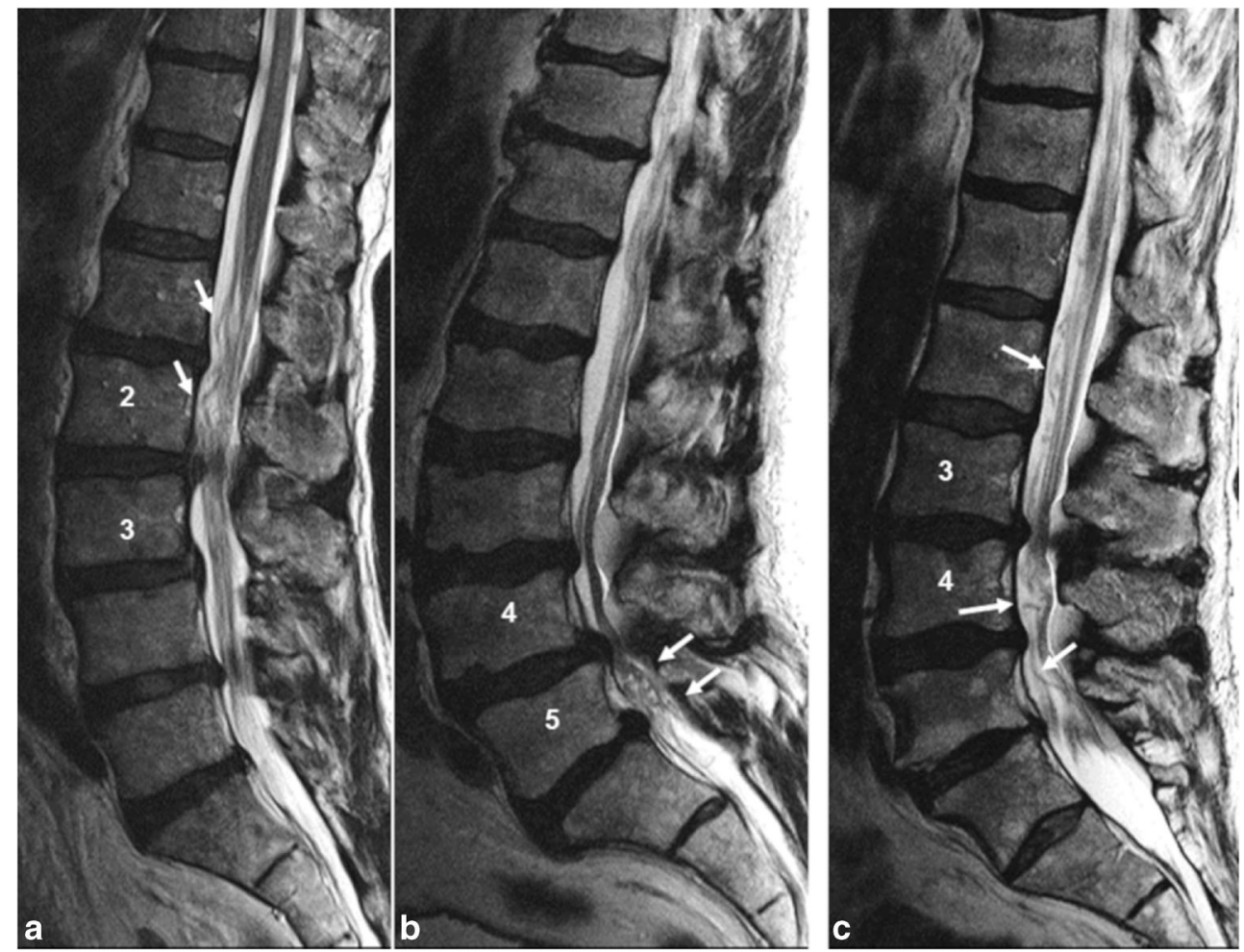


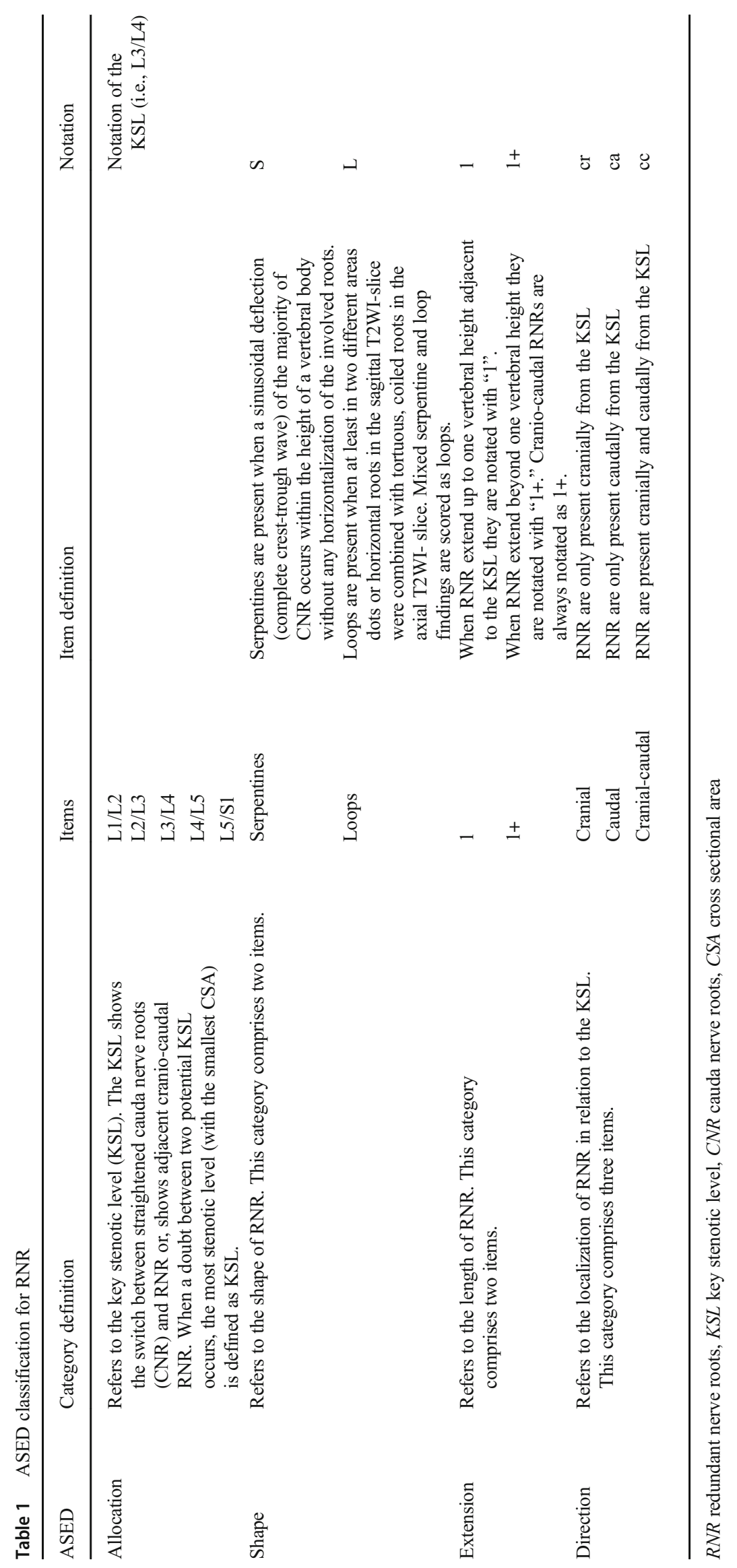


previously agreed KSL allocations. In the second read, performed 4 weeks later, after altering the order of cases, the allocation values were used from the first read. These were fixed after calculating the inter-rater kappa values. Because the allocation category was only rated once, intra-rater kappa values for this category were not calculated.

In addition to the ASED classification, all raters classified the LSS grade with the qualitative grading system, based on the root-to-cerebrospinal fluid relationship described by Schizas et al. [17].

\section{Statistical analysis}

To determine the proportion of agreement, the Fleiss kappa $(k)$ was used to assess inter-rater reliability. The Fleiss kappa is an extension of Cohen's kappa; it can be used when nominal categories are assessed by more than two raters [18]. In this study, Fleiss kappa values were calculated for junior raters, senior raters, and for all 6 raters, for both reads. Cohen's kappa $(k)$ was used to calculate intra-rater reliability [19]. Mean kappa values for intra-rater reliability were calculated separately for junior and senior raters. The $k$ values were categorized to reflect different levels of agreement, as follows: $\leq 0.00$ : poor, 0.00-0.20: slight, $0.21-0.40$ : fair, $0.41-0.60$ : moderate, $0.61-$ 0.80 : substantial, and $\geq 0.81$ : almost perfect [20].

To determine whether inter-rater kappa values differed significantly between the two reads, we performed paired sample $t$ tests. Inter-rater kappa values for the 1 st and 2 nd reads were compared between junior and senior raters with independent sample $t$ tests. The assumptions associated with the different tests were verified previously.

We performed all statistical analyses with IBM SPSS software version 21 for Macintosh (IBM Corp. Armonk, New York). The 0.05 level of probability was set as the criterion for statistical significance.

\section{Results}

The results for inter-rater reliability are presented in Table 2. The ASED classification showed moderate-toalmost perfect inter-rater reliability. In the 1st read, all 6 raters achieved kappa values $(95 \% \mathrm{CI})$ that ranged from $0.56(0.51,0.60)$, for extension, to $0.86(0.83,0.90)$, for allocation. The kappa values of junior raters did not change significantly between the 1 st and the 2 nd reads $(P=0.06)$. In contrast, senior raters achieved higher inter-rater kappa values in the 2 nd read than in the first read $(P=0.008)$. When all raters $(n=6)$ were considered, there was no significant difference between interrater kappa values of both reads $(P=0.5)$.

The results for intra-rater reliability are presented in Table 3. The ASED classification showed almost perfect intra-rater reliability. For junior raters, the mean kappa values ranged from $0.83(0.76,0.90)$, for shape, to 0.86 $(0.82,0.90)$ for extension. Senior raters achieved similar mean kappa values, except in the shape category $(k=$ $0.90[0.88,0.92])$.

Inter-rater reliability for LSS grade was substantial in the first read for all raters $(k=0.69[0.65,0.74])$. The intra-rater reliabilities were substantial $(k=0.78[0.67,0.89])$ and almost perfect $(k=0.88[0.83,0.93])$, for senior and junior raters, respectively.

In $95.6 \%$ of cases, the KSL was located in the central part of the lumbar spine, with $n=56(44.4 \%)$ at L3/L4, $n=35$ $(27.8 \%)$ at $\mathrm{L} 4 / \mathrm{L} 5$, and $n=31(24.6 \%)$ at $\mathrm{L} 2 / \mathrm{L} 3$. In four cases (3.2\%), the KSL was located at L1/L2, but it was never located at $\mathrm{L} 5 / \mathrm{S} 1$.

The severity of LSS was scored according to the classification purposed by Schizas et al. [17]. We observed "surgical" grade C in $94(75 \%)$ cases and grade $\mathrm{D}$ in $30(24 \%)$ cases. We observed stenosis grade B in two cases $(1 \%)$.

Table 2 Inter-rater reliability for the ASED classification of RNR

\begin{tabular}{|c|c|c|c|c|c|c|}
\hline \multirow[t]{2}{*}{ ASED categories/items } & \multicolumn{3}{|l|}{1 st read } & \multicolumn{3}{|l|}{ 2nd read } \\
\hline & $\begin{array}{l}\text { Junior raters }(n=3) \\
\text { Kappa }(95 \% \text { CI })\end{array}$ & $\begin{array}{l}\text { Senior raters }(n=3) \\
\text { Kappa }(95 \% \text { CI })\end{array}$ & $\begin{array}{l}\text { All raters }(n=6) \\
\text { Kappa }(95 \% \text { CI })\end{array}$ & $\begin{array}{l}\text { Junior raters }(n=3) \\
\text { Kappa }(95 \% \text { CI })\end{array}$ & $\begin{array}{l}\text { Senior raters }(n=3) \\
\text { Kappa }(95 \% \mathrm{CI})\end{array}$ & $\begin{array}{l}\text { All raters }(n=6) \\
\text { Kappa }(95 \% \text { CI })\end{array}$ \\
\hline Allocation & $0.89(0.82,0.96)$ & $0.82(0.70,0.94)$ & $0.86(0.83,0.90)$ & $0.89(0.82,0.96)$ & $0.82(0.70,0.94)$ & $0.86(0.83,0.90)$ \\
\hline Shape & $0.66(0.56,0.76)$ & $0.62(0.52,0.72)$ & $0.62(0.57,0.66)$ & $0.59(0.49,0.69)$ & $0.63(0.53,0.73)$ & $0.59(0.55,0.64)$ \\
\hline Extension & $0.57(0.47,0.67)$ & $0.60(0.49,0.70)$ & $0.56(0.51,0.60)$ & $0.53(0.43,0.63)$ & $0.68(0.58,0.78)$ & $0.59(0.55,0.64)$ \\
\hline Direction (overall) & $0.64(0.57,0.72)$ & $0.74(0.64,0.82)$ & $0.66(0.63,0.70)$ & $0.62(0.55,0.70)$ & $0.82(0.74,0.90)$ & $0.65(0.62,0.69)$ \\
\hline Cranial (cr) & $0.74(0.64,0.84)$ & $0.80(0.70,0.90)$ & $0.76(0.72,0.81)$ & $0.68(0.58,0.78)$ & $0.89(0.79,1)$ & $0.75(0.71,0.80)$ \\
\hline Caudal (ca) & $0.67(0.56,0.77)$ & $0.80(0.70,0.91)$ & $0.72(0.67,0.76)$ & $0.63(0.53,0.73)$ & $0.83(0.73,0.93)$ & $0.68(0.63,0.72)$ \\
\hline Cranio-caudal (cc) & $0.48(0.37,0.58)$ & $0.38(0.28,0.48)$ & $0.39(0.35,0.44)$ & $0.53(0.43,0.63)$ & $0.54(0.44,0.64)$ & $0.42(0.38,0.47)$ \\
\hline LSS grade ${ }^{\mathrm{a}}$ & $0.77(0.67,0.87)$ & $0.64(0.54,0.74)$ & $0.69(0.65,0.74)$ & $0.68(0.58,0.78)$ & $0.76(0.67,0.86)$ & $0.67(0.62,0.71)$ \\
\hline
\end{tabular}

Values are Fleiss kappa with 95\% confidence intervals for junior raters, senior raters, and for all six raters, for the 1st and 2nd reads

${ }^{\text {a }}$ Grade of LSS according to Schizas et al. [17] 


\section{Discussion}

Previous reports have shown that patients with LSS that displayed RNRs in preoperative MRIs had worse postoperative outcomes compared to patients without evidence of RNRs. Those findings suggested that RNRs comprise a negative prognostic factor $[9,11,21]$.

To the best of our knowledge, a validated MRI-based RNR classification has not been established. Here, we presented an ASED classification for RNRs. Six raters with different grades of experience independently scored 126 MRIs with ASED classifications. They exhibited moderate to almost perfect inter-rater and almost perfect intra-rater reliabilities. These results indicated that the ASED classification could be used in daily radiological practice to complete MRI reports on patients with LSS.

We classified the LSS severity grade with the well-known grading system of Schizas et al. [17]. They assessed its intraand inter-rater reliabilities with 57 axial T2 MRIs of patients with LSS. Those authors reported average kappa values of $0.44 \pm 0.17$ and $0.65 \pm 0.14$ for inter- and intra-rater reliabilities, respectively. Raters from the originating study unit achieved higher kappa values $(k=0.67 \pm 0.08$ and $k=0.77$ \pm 0.06 , respectively). Our study results confirmed their results with a sample that was twofold larger; we found $k=0.69$ and $k=0.78$ for inter- and intra-rater reliabilities, respectively (Tables 2 and 3). Moreover, like Schizas et al., we also found that inter-rater kappa values were lower than intra-rater kappa values. However, in their study, neither inter-rater nor intrarater reliability achieved mean kappa values above substantial agreement.

The ASED classification comprised four categories with 12 items for scoring RNRs on T2 sagittal and axial MRIs. This classification is more complex than the LSS grading system [17]. Our results indicated that the ASED was reliable and could be used in clinical practice.

In the 1st read, the inter-rater kappa values of all 6 raters for the direction category were $0.76(0.72,0.81)$ for cranial, 0.72 $(0.67,0.76)$ for caudal, and $0.39(0.35,0.44)$ for cranio-caudal. However, kappa values are affected by the distribution of data across the categories (prevalence bias). The frequency distribution that we observed across the three items in the direction category was $n=84(66.7 \%)$ for cranial, $n=35$ (27.8\%) for caudal, and $n=7$ (5.6\%) for cranio-caudal. This unequal distribution influenced the kappa values of the respective items, as outlined previously by Byrt et al. [22].

Our results confirmed the surgical relevance of the KSL, the key element of the ASED classification. When rating the $\mathrm{KSL}$, the raters were blinded to the surgical levels. Interestingly, all except four KSLs (97\%) were decompressed. Moreover, in 42 patients (33\%), a second level was decompressed, and in eight other patients (6\%), two additional levels were decompressed. Of the four cases that showed a 
discrepancy between the KSL and the operated level, two patients displayed more stenosis at the operated level than at the KSL, and the two other patients displayed disc herniations associated with a stenotic level adjacent to the KSL.

In our daily practice, we nicknamed the KSL "the switch level," because, in 119 out of 126 patients, at the KSL, the CNR "switched" from a straight course to a RNR shape. In the other seven patients, the KSL was embedded in a craniocaudal RNR.

In addition, we confirmed the clinical relevance of the LSS grade classification of Schizas et al. [17]. We observed "surgical" grade C in 94 (75\%) patients and grade D in 30 (24\%) patients.

Physicians that care for patients with LSS expect the MRI report to answer the following questions: Is there any LSS, and how severe is it? Which level(s) and anatomic structures are involved? In our opinion, to counsel patients with LSS about adequate treatment, clinicians also need information about the degree of compromise at the CNR. Thus, the MRI report should answer the question: Are RNRs present, and what is their shape, extension, and direction? A previous study by Min et al. [23] examined associations between the relative length of RNRs and the symptom duration and recovery rates; they found moderately positive $(r=0.38)$ and strongly positive ( $r=0.53)$ correlations, respectively. Ono et al. [4] reported that a group with higher numbers of loop RNRs had a higher mean duration of neurological symptoms and worse preoperative walking ability, compared to a group with higher numbers of serpentine RNRs. To investigate these issues further, a validated RNR classification system is necessary.

In a previous review, Nogueira-Barbosa et al. [24] suggested that radiologists should examine MRIs for RNRs in the cauda equina and, when applicable, describe those findings in the MRI report. We share that opinion, and to facilitate the descriptions, we have presented the ASED classification.

Although imaging should not influence the surgical indication $[12,25]$, our results pointed out the relevance of imaging in surgical planning. We defined the MRI quality of the sample by choosing a field strength of at least 1.5 T. However, in daily practice, different observers have different perceptions of the image quality of $1.5 \mathrm{~T}$ MRIs.

For a long time, researchers have debated the validity of the signal-to-noise ratio as an objective quality measure for biomedical images [26]. In the present study, different image resolutions may have led to differences in scoring. This was a study limitation.

In conclusion, we demonstrated that the ASED classification for RNRs was reliable and feasible. It should be included in the MRI report for patients with LSS that display evidence of RNRs.

Acknowledgments The authors thank former patient Mr. Dieter Witt for sponsoring Open Access .
Funding information This study received no funding.

\section{Compliance with ethical standards}

Conflict of interest The authors declare that they have no conflict of interest.

Ethical approval All procedures were in accordance with the ethical standards of the Ethic Committee of the Federal State of Hamburg, Germany.

Informed consent For this type of study, formal consent is not required.

Open Access This article is licensed under a Creative Commons Attribution 4.0 International License, which permits use, sharing, adaptation, distribution and reproduction in any medium or format, as long as you give appropriate credit to the original author(s) and the source, provide a link to the Creative Commons licence, and indicate if changes were made. The images or other third party material in this article are included in the article's Creative Commons licence, unless indicated otherwise in a credit line to the material. If material is not included in the article's Creative Commons licence and your intended use is not permitted by statutory regulation or exceeds the permitted use, you will need to obtain permission directly from the copyright holder. To view a copy of this licence, visit http://creativecommons.org/licenses/by/4.0/.

\section{References}

1. Deyo RA (2010) Treatment of lumbar spinal stenosis: a balancing act. Spine J 10(7):625-627. https://doi.org/10.1016/j.spinee.2010. 05.006

2. Chen J, Wang J, Wang B, Xu H, Lin S, Zhang H (2016) Postsurgical functional recovery, lumbar lordosis, and range of motion associated with MR-detectable redundant nerve roots in lumbar spinal stenosis. Clin Neurol Neurosurg 140:79-84. https://doi.org/ 10.1016/j.clineuro.2015.11.016

3. Hur JW, Hur JK, Kwon TH, Park YK, Chung HS, Kim JH (2012) Radiological significance of ligamentum flavum hypertrophy in the occurrence of redundant nerve roots of central lumbar spinal stenosis. J Korean Neurosurg Soc 52(3):215-220. https://doi.org/10. 3340/jkns.2012.52.3.215

4. Min JH, Jang JS, Lee SH (2008) Clinical significance of redundant nerve roots of the cauda equina in lumbar spinal stenosis. Clin Neurol Neurosurg 110(1):14-18. https://doi.org/10.1016/j. clineuro.2007.08.005

5. Savarese LG, Ferreira-Neto GD, Herrero CF, Defino HL, NogueiraBarbosa MH (2014) Cauda equina redundant nerve roots are associated to the degree of spinal stenosis and to spondylolisthesis. Arq Neuropsiquiatr 72(10):782-787

6. Verbiest H (1954) A radicular syndrome from developmental narrowing of the lumbar vertebral canal. J Bone Joint Surg Br 36$\mathrm{B}(2): 230-237$

7. Cressman MR, Pawl RP (1968) Serpentine myelographic defect caused by a redundant nerve root. Case report. J Neurosurg 28(4): 391-393. https://doi.org/10.3171/jns.1968.28.4.0391

8. Banse X, Manil O, Lecouvet F, Duprez T (2010) Roots trajectory abnormalities and stenosis severity. Eur Spine J 19(Suppl 3):311312

9. Marques CJ, Hillebrand H, Papavero L (2018) The clinical significance of redundant nerve roots of the cauda equina in lumbar spinal stenosis patients: a systematic literature review and meta-analysis. 
Clin Neurol Neurosurg 174:40-47. https://doi.org/10.1016/j. clineuro.2018.09.001

10. Papavero L, Marques CJ, Lohmann J, Fitting T (2018) Patient demographics and MRI-based measurements predict redundant nerve roots in lumbar spinal stenosis: a retrospective database cohort comparison. BMC Musculoskelet Disord 19(1):452. https://doi.org/10. 1186/s12891-018-2364-4

11. Yokoyama K, Kawanishi M, Yamada M, Tanaka H, Ito Y, Hirano M, Kawabata S, Kuroiwa T (2014) Clinical significance of postoperative changes in redundant nerve roots after decompressive laminectomy for lumbar spinal canal stenosis. World Neurosurg 82(6):e825-e830. https://doi.org/10.1016/j.wneu.2013.09.024

12. Andreisek G, Imhof M, Wertli M, Winklhofer S, Pfirrmann CW, Hodler J, Steurer J (2013) A systematic review of semiquantitative and qualitative radiologic criteria for the diagnosis of lumbar spinal stenosis. AJR Am J Roentgenol 201(5):W735-W746. https://doi. org/10.2214/AJR.12.10163

13. Kottner J, Audige L, Brorson S, Donner A, Gajewski BJ, Hrobjartsson A, Roberts C, Shoukri M, Streiner DL (2011) Guidelines for Reporting Reliability and Agreement Studies (GRRAS) were proposed. Int J Nurs Stud 48(6):661-671. https:// doi.org/10.1016/j.jinurstu.2011.01.016

14. von Elm E, Altman DG, Egger M, Pocock SJ, Gotzsche PC, Vandenbroucke JP, Initiative S (2007) The Strengthening the Reporting of Observational Studies in Epidemiology (STROBE) statement: guidelines for reporting observational studies. Lancet 370(9596):1453-1457. https://doi.org/10.1016/S0140-6736(07) 61602-X

15. Rotondi MA, Donner A (2012) A confidence interval approach to sample size estimation for interobserver agreement studies with multiple raters and outcomes. J Clin Epidemiol 65(7):778-784. https://doi.org/10.1016/j.jclinepi.2011.10.019

16. Poureisa M, Daghighi MH, Eftekhari P, Bookani KR, Fouladi DF (2015) Redundant nerve roots of the cauda equina in lumbar spinal canal stenosis, an MR study on 500 cases. Eur Spine J 24(10): 2315-2320. https://doi.org/10.1007/s00586-015-4059-y

17. Schizas C, Theumann N, Burn A, Tansey R, Wardlaw D, Smith FW, Kulik G (2010) Qualitative grading of severity of lumbar spinal stenosis based on the morphology of the dural sac on magnetic resonance images. Spine (Phila Pa 1976) 35(21):1919-1924. https://doi.org/10.1097/BRS.0b013e3181d359bd

18. Fleiss JL (1971) Measuring nominal scale agreement among many raters. Psychol Bull 76(5):378-382. https://doi.org/10.1037/ h0031619

19. Cohen J (1960) A coefficient of agreement for nominal scales. Educ Psychol Meas 20(1):37-46. https://doi.org/10.1177/ 001316446002000104

20. Landis JR, Koch GG (1977) The measurement of observer agreement for categorical data. Biometrics 33(1):159-174

21. Cong L, Zhu Y, Yan Q, Tu G (2017) A meta-analysis on the clinical significance of redundant nerve roots in symptomatic lumbar spinal stenosis. World Neurosurg 105:95-101. https://doi.org/10.1016/j. wneu.2017.05.103

22. Byrt T, Bishop J, Carlin JB (1993) Bias, prevalence and kappa. J Clin Epidemiol 46(5):423-429

23. Ono A, Suetsuna F, Irie T, Yokoyama $T$, Numasawa $T$, Wada $K$, Toh S (2007) Clinical significance of the redundant nerve roots of the cauda equina documented on magnetic resonance imaging. J Neurosurg Spine 7(1):27-32. https://doi.org/10.3171/SPI-07/07/ 027

24. Nogueira-Barbosa MH, Savarese LG, Herrero CFPS, Defino HLA (2012) Redundant nerve roots of the cauda equina: review of the literature. Radiol Bras 45(3):155-159

25. Mamisch N, Brumann M, Hodler J, Held U, Brunner F, Steurer J (2012) Radiologic criteria for the diagnosis of spinal stenosis: results of a Delphi survey. Radiology 264(1):174-179. https://doi. org/10.1148/radiol.12111930

26. Erdogmus D, Larsson EG, Yan R, Principe JC, Fitzsimmons JR (2004) Measuring the signal-to-noise ratio in magnetic resonance imaging: a caveat. Signal Process 84(6):1035-1040. https://doi.org/ 10.1016/j.sigpro.2004.03.006

Publisher's note Springer Nature remains neutral with regard to jurisdictional claims in published maps and institutional affiliations. 